\title{
Editorial
}

\section{Cellular cardiomyoplasty: a new hope in heart failure?}

Despite recent advances in the management of patients with heart failure, and because of the scarcity of heart donors, the incidence and prevalence of the disease remains notably high in our countries. Recent epidemiological data have shown an incidence of 225 patients with severe heart failure per million, with a rate of death of $35 \%$ per year. ${ }^{1}$ This has encouraged the development of new methods of biological assistance, also called cardiomyoplasty techniques. The first of these is dynamic cardiomyoplasty, which uses the latissimus dorsi muscle wrapped around a deficient heart which is stimulated, but this procedure produces inconsistent and moderate objective haemodynamic effects. Another technique is molecular cardiomyoplasty, which is based on the transformation of non-myogenic into contractile cells or attempts to induce the cardiomyocytes to re-enter the cellular cycle; for the moment this remains out of reach. A third technique is cellular cardiomyoplasty, which involves myogenic cell grafting within the myocardium to limit any consequences of the loss of contractile function of a damaged left ventricle. $^{2}$

\section{Somatic cell transplantation}

Transplantation of somatic cells to supply the function of a deficient organ has been successfully performed for decades for bone marrow, and more recently, only with inconsistent results, for skeletal muscles (Duchenne dystrophy), liver (as a bridge to transplantation), pancreas (islets of Langerhans) or brain. ${ }^{2}$ It has been shown that minced fetal brain tissue can be grafted within the brain of Parkinsonian patients, increasing dopamine secretion and decreasing symptoms. Like brain cells, adult ventricular myocytes are terminally differentiated, with no possibility of cell division from neonatal age; thus, following injury (infarction), repair consists of scar formation, hypertrophy of surviving myocytes, and hyperplasia of non-muscle cells such as fibroblasts (remodelling). These phenomena become deleterious over time and do not address the initial problem, the loss of contractile cells. Thus, as with Parkinson's disease, cultured fetal cells have been first used as intramyocardial grafts.

\section{Intramyocardial grafting}

\section{IN VITRO STUDIES}

In vitro studies in animals have shown that transplanted cells within a normal or infarcted myocardium remain viable for months and can proliferate and differentiate in $\mathrm{situ}^{3}$; the presence of intercalated disks and connexin 43, a marker of gap junctions required for cell-to-cell electrical coupling, has been demonstrated within grafted tissue and between grafted cells and host myocytes in the case of fetal cell transplantation. ${ }^{45}$ The graft can demonstrate contraction (spontaneously in the case of fetal cells or after electrical stimulation for the myoblasts), induces angiogenesiswhich is beneficial in case of ischaemic heart disease-and local immune reactions which are responsible for the rapid death (necrosis, apoptosis) of a variable number of the allografted cells. Finally, the beneficial influence of the procedure on left ventricular performance has been suggested after cryoinfarction in rats using a Langendorff model; when compared to controls, transplanted hearts showed no infarct expansion, presence of neo-tissue (which occupied more than one third of the scar), and a higher developed systolic pressure. ${ }^{6}$

IN VIVO EXPERIMENTAL STUDIES

Intramyocardial injections of cultured fetal cardiomyocytes after infarction in female rats in an ischaemia-reperfusion model increased ejection fraction at one month; male cells transplanted into the female hosts were identified at necropsy by detection of the $\mathrm{Y}$ chromosome using an in situ hybridisation technique. ${ }^{7}$ In a mouse model of doxorubicin induced global cardiomyopathy, ${ }^{8}$ local transplantation of fetal cardiomyocytes can also improve global function, suggesting the intervention of paracrine factors. Comparing intramyocardial injections of cultured fetal cardiomyocytes and allogenic fetal skeletal myoblasts after infarction in rats, ${ }^{9}$ the functional equivalence of these two types of cells was demonstrated with decreased cavity dilatation and increased ejection fraction of similar magnitude; this "anti-remodelling" effect was more pronounced for the lowest ejection fractions $(<40 \%)$ with an approximately $30 \%$ relative increase in ejection fraction without cavity dilatation. Similar results were obtained with cultured autologous myoblasts from the tibialis anterior injected directly into the myocardium of rats, and viable myoblastic tissue was observed at histology with confluence into myotubes ${ }^{10}$; the presence of connexin 43 has been found in vitro (between grafted myoblasts and between donor and recipient cells), but inconsistently in vivo. ${ }^{911}$ However, such a coupling is suggested by the fact that the greater the quantity of myoblasts injected, the greater the increase in ejection fraction. ${ }^{10}$ However, contrary to what occurs after transplantation of fetal tissue in Parkinsonian patients, use of minced skeletal muscle has no effect. ${ }^{10}$ Other authors found similar beneficial functional results with autologous myoblasts grafted after infarction in the rabbit using a conductance catheter technique or pressure volume loop tracings, and in the dog using micromanometers and epicardial microcrystals. ${ }^{11-13}$ These findings have been reported along with a decrease in infarct size.

Which are the optimal cells?

The aim is to obtain the highest number of myogenic cells for transplantation, their culture being an essential step. Thus, these cells must possess a high potential for division during culture, and ideally have a potential for further multiplication after grafting. Immortalised cultured myogenic cell lines have been used experimentally but there is a potential for cancer which limits a potential human use. Fetal cardiomyocytes - which can divide normally - have been successfully grafted into the myocardium, but their use raises immunological, ethical, and availability problems. Thus, it appears that cultured autologous myogenic cells are an optimal support, as they obviate the need for immunosuppressive treatment. Skeletal myoblasts (also called satellite cells and functionally indistinguishable from embryonic myoblasts) are stem cells located at the basal 
lamina of the adult skeletal muscle, are highly resistant to ischaemia, and may multiply after injury (each myoblast can supply about 12 mitoses); this explains why iatrogenic rhabdomyolysis can be followed by a restitution ad integrum after removal of the causal drug. From a clinical perspective, the use of cultured autologous myoblasts requires a strict protection against bacterial, viral or prion contamination, and a high efficacy of cell culture. We have now developed techniques which enable us to obtain hundreds of thousands of myoblasts from a single human muscular biopsy weighing a few grams (authors' unpublished data). Bone marrow stem cells are also able to differentiate into myogenic cells, ${ }^{14}$ but their clinical use remains hypothetical. In the future, adjunctive treatments could include ex vivo genetic engineering targeted at preventing apoptosis or increasing angiogenesis; moreover, cells might be delivered using intracardiac catheters instead of thoracotomy.

\section{Unresolved questions}

The mechanisms of these beneficial effects remain controversial. It is not certain whether improvement in left ventricular performance is mediated by an increased systolic function caused by synchronous contraction of the graft. An indirect systolic effect - mediated by angiogenic or growth factors secreted by transplanted cells-is suggested by the improvement of global function of locally injected cells. ${ }^{8}$ A passive girdling effect, simply limiting progressive cavity dilatation and decreasing pump function, is likely. Increased myocardial thickness caused by local injection may decrease wall stress, thus improving function and reducing infarct expansion. Recent experimental studies have suggested a direct systolic effect (increased systolic thickening) of myogenic cell transplantation in the infarcted area. ${ }^{11}$ It is noteworthy, however, that a significant improvement in function requires transplantation of contractile cells, as intramyocardially grafted fibroblasts are far less effective.

\section{From bench to bedside}

Thus, cellular transplantation into a damaged myocardium results in an obvious improvement in left ventricular performance and remodelling. Experiments have shown a similar efficacy of fetal and satellite cells, using either allogenic or preferably autologous grafts which do not raise any immunological, ethical, tumorigenesis, or donor availability problems; this opens the way to human trials in the near future. The first clinical applications should ideally be the postinfarction patient with a low ejection fraction and an indication for coronary artery bypass surgery. After skeletal muscular biopsy, cultured autologous myoblasts could be directly administered by multiple injections within and around the infarcted area during surgery. The first step will be to check the feasibility of the whole procedure (from biopsy to reinjections after large scale cell expansion) and its safety (absence of induced arrhythmias); its efficacy, which is suggested by a wealth of experimental data in different species using multiple evaluation techniques, will then have to be clinically proven.

Faculté de Médecine Necker Enfants-Malades,

ALBERT A HAGÈGE

Université Paris $V$

Department of Cardiology 3,

Hôpital Européen Georges Pompidou,

Paris, France

Department of Cardiac Surgery B,

PHILIPPE MENASCHÉ

Hôpital Bichat,

Paris, France

Correpondence to: Dr Hagège

hagege@club-internet.fr

1 Zannad F, Briançon S, Juilliere Y, et al. Incidence, clinical and etiologic features, and outcomes of advanced chronic heart failure: the EPICAL study. 7 Am Coll Cardiol 1999;33:734-42.

2 Hagège AA, Scorsin M, Samuel JL, et al. Cellular cardiomyoplasty: state of the art, evaluation and future possibilities. Arch Mal Coeur 1998;91:142935 .

3 Scorsin M, Marotte F, Sabri A, et al. Can grafted cardiomyocytes colonize periinfarction myocardial areas ? Circulation 1996;94(suppl II):II-337-40. 4 Soonpaa $\mathrm{MH}$, Koh GY, Klug MG, et al. Formation of nascent intercalated disks between grafted fetal cardiomyocytes and host myocardium. Science 1994;264:98-101.

5 Reinecke H, Zhang M, Bartosek T, et al. Survival, integration, and differentiation of cardiomyocyte grafts: a study in normal and injured rat hearts. Circulation 1999;100:193-202.

6 Li RK, Jia ZQ, Weisel RD, et al. Cardiomyocyte transplantation improves heart function. Ann Thorac Surg 1996;62:654-61.

7 Scorsin $\mathrm{M}$, Hagège AA, Marotte F, et al. Does transplantation of cardiomyocytes improve function of infarcted myocardium ? Circulation 1997;96(suppl II):II-188-93.

8 Scorsin M, Hagège AA, Dolizy I, et al. Can cellular transplantation improve function in doxorubicin-induced heart failure? Circulation 1998;98 (suppl function in $151-6$.

9 Scorsin M, Hagège A, Vilquin JT, et al. Comparison of the effects of fetal cardiomyocytes and skeletal myoblasts transplantation on post-infarct left cardiomyocytes and skeletal myoblasts transplantation on post-inf
ventricular function. $\mathcal{F}$ Thorac Cardiovasc Surg 2000;119:1169-75.

10 Pouzet B, Vilquin JT, Hagège A, et al. Intramyocardial transplantation of autologous myoblasts: can tissue processing be optimized? Circulation In press.

11 Kao RL, Chin TK, Ganote CE, et al. Satellite cell transplantation to repair injured myocardium. Cardiac and Vascular Regeneration 2000;1:31-42.

12 Taylor DA, Atkins ZB, Hungspreugs P, et al. Regenerating functional myocardium: improved performance after skeletal myoblast transplantation. Nature Med 1998;4:929-33.

13 Atkins ZB, Hueman MT, Meuchel J, et al. Cellular cardiomyoplasty improved diastolic properties of injured heart. F Surg Res 1999;85:234-42.

14 Wakitani S, Saito T, Caplan AI. Myogenic cells derived from rat bone marrow mesenchymal stem cells exposed to 5-azacytidine. Muscle Nerve 1995; 18:1417-26. 